\title{
Finite-size effect on the resistive state in a mesoscopic type-II superconducting stripe
}

\author{
G. R. Berdiyorov, A. K. Elmurodov, and F. M. Peeters* \\ Department of Physics, University of Antwerp, Groenenborgerlaan 171, B-2020 Antwerpen, Belgium \\ D. Y. Vodolazov \\ Department of Physics, Nizhny Novgorod University, 603600 Nizhny Novgorod, Russia
}

(Received 26 December 2008; published 5 May 2009)

\begin{abstract}
Within the time-dependent Ginzburg-Landau (TDGL) theory we studied the creation of phase-slip lines and the interplay with a vortex lattice in a finite-length superconducting thin stripe with finite-size normal metal leads. In zero magnetic field and with increasing transport current phase-slip lines appear across the sample leading to distinct jumps in the current-voltage characteristics. When a magnetic field is applied, the moving vortex lattice becomes rearranged by the external current and fast and slow moving vortex channels are formed. Curved vortex channels are observed near the normal contacts. We found the remarkable result that at small applied magnetic field the normal-state transition current is increased as compared to the one at zero magnetic field. This effect is more pronounced for larger values of the $\gamma$ parameter in the TDGL formalism. This unusual "field-induced" increase in the critical current is a consequence of the nonuniform distribution of the current in the sample.
\end{abstract}

DOI: $10.1103 /$ PhysRevB.79.174506

PACS number(s): 74.20.De, 74.25.Dw, 74.78.Na, 74.25.Ha

\section{INTRODUCTION}

When a thin superconducting film is placed in a perpendicular magnetic field that is larger than some critical value, Abrikosov vortices penetrate the sample and form a triangular vortex lattice in the absence of pinning. If now a transport current is applied to the sample vortices start moving under the action of the Lorentz force of the current. The vortex motion leads to energy dissipation in the system and to a finite voltage and electrical field in the superconductor. At large vortex line velocities, the vortex motion becomes unstable and a nonequilibrium distribution of the quasiparticles appear due to the slow energy relaxation, which leads to an electronic instability and an abrupt switching into a state with higher electrical resistivity, i.e., the voltage-current characteristic exhibits a hysteretic jump, as predicted by Larkin and Ovchinnikov (LO). ${ }^{1}$ The induced electric field due to vortex motion results in a decreasing size of the vortex cores because quasiparticles accelerated by the electrical field can reach energies above the superconducting energy gap and diffuse away from the vortex core.

Electric-field-induced flux flow instabilities, intensively studied in the past both in low-temperature ${ }^{2-5}$ and high-temperature ${ }^{6-10}$ superconductors, in general agree reasonably well with the LO theory. However, in some cases, the LO theory turns out to be insufficient to describe the experimental results. For example, at low temperatures the LO description breaks down, suggesting a different origin for the flux flow instabilities. ${ }^{4}$ Explanation for the flux flow instabilities beyond the original or modified LO picture was sought in dynamic vortex lattice crystallization, ${ }^{11}$ depinning phenomena, ${ }^{12}$ appearance of hot spots, ${ }^{13}$ and recently in vortex core expansion due to electron heating at low temperatures. ${ }^{14}$

However, in the original LO theory the question about vortex structure before and after the transition was not considered. At the end of the 1970s it was speculated that lines with fast vortex motion [so-called phase-slip lines (PSLs)] should appear in the superconductor at the transition point. Because analytical calculations are strongly restricted due to the mathematical complexity of the problem, only a semiquantitative analysis was made using the assumption that phase-slip lines already exist in the sample. ${ }^{15}$

A number of works have been published where vortex motion was studied theoretically using a numerical simulation of the extended time-dependent Ginzburg-Landau (TDGL) equations ${ }^{16-19}$ where the time $\tau_{\text {in }}$ of the nonequilibrium quasiparticle distribution function was explicitly included. In Refs. 16 and 17 two types of vortex motion were found: slow and fast vortex motions (the latter was named the kinematic vortex ${ }^{17}$ ). However, neither the influence of the magnetic field nor the transition between these types of motion nor the transformation of the vortex lattice were addressed. In Ref. 18 the deformation of the vortex core due to the finite relaxation time of the order parameter was found and a short-range attraction between vortices was predicted. In a recent paper ${ }^{19}$ the rearrangement of the vortex lattice due to the above effect and the transition from the slow to the fast vortex motion (phase-slip line) were studied for an infinitely long superconducting slab placed in a parallel magnetic field.

In most of the experiments devoted to the study of transport properties of superconducting samples, a microbridge geometry is used in order to reduce the power dissipation in the system. However, in the presence of an external magnetic field the bulk superconducting leads may turn into a normal state while the microbridge is still superconducting, which considerably changes the experimental picture. For example, the critical current of the superconducting nanowire can be significantly enhanced, ${ }^{20}$ as well as the critical current for the creation of phase-slip centers can be affected ${ }^{21}$ by the superconducting normal transition of the leads. Thus, in some of the cases we have to deal with normal contacts. The presence of normal electrodes is in general closer to the real experimental situation, which may change the properties of the whole system in comparison with the solitary mesoscopic 


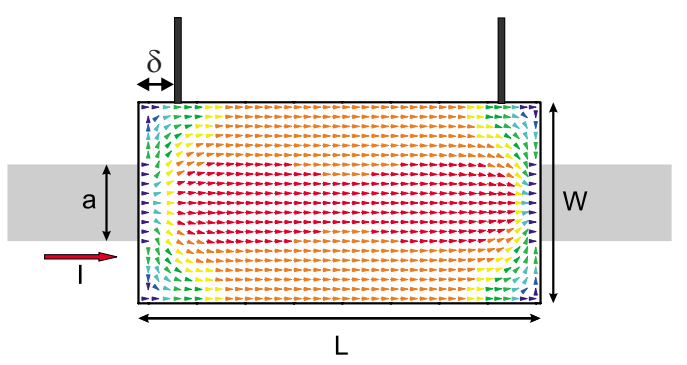

FIG. 1. (Color online) A schematic view of the studied superconducting strip with attached normal leads. Vertical black stripes show the position of potential contacts. Vector plot shows the distribution of the superconducting current in the sample in case of $H=0$ and nonzero applied transport current. Red (larger arrows) or blue (smaller arrows) regions correspond to high or low current.

origin due to also possible quantum interference.

In this paper we investigate nonequilibrium processes in a superconducting stripe in the presence of a perpendicular magnetic field and a transport current. The effect of the finite size of the sample as well as the effect of the normal contacts on the current-voltage $(I-V)$ characteristics of the sample are considered. Due to the finite length of the strip and the intrinsically nonuniform current distribution along the sample (because of normal current contacts-see Fig. 1) the system is different from the one of Ref. 19, where periodic boundary conditions were assumed and thus an infinite long sample was studied.

\section{THEORETICAL FORMALISM}

We consider a very thin (thickness $d \ll \xi, \lambda$ ) superconducting strip (length $L$ and width $W$ ) in the presence of a perpendicular magnetic field with a transport electric current applied through the normal contacts (size $a$ ) (see Fig. 1). In the case of thin films (with $W<\lambda^{2} / d$ ) the self-induced magnetic field can be neglected (weak demagnetization effect) and the generalized TDGL equation averaged over the sample thickness $^{22}$ can be written in the following form: ${ }^{23,24}$

$$
\begin{aligned}
& \frac{u}{\sqrt{1+\gamma^{2}|\psi|^{2}}}\left(\frac{\partial}{\partial t}+i \varphi+\frac{\gamma^{2}}{2} \frac{\partial|\psi|^{2}}{\partial t}\right) \psi=(\nabla-i \mathbf{A})^{2} \psi+(1 \\
& \left.-|\psi|^{2}\right) \psi
\end{aligned}
$$

This equation should be supplemented with the equation for the electrostatic potential

$$
\Delta \varphi=\operatorname{div}\left\{\operatorname{Im}\left[\psi^{*}(\nabla-i \mathbf{A}) \psi\right]\right\},
$$

which is nothing else than the condition for the conservation of the total current in the wire, i.e., div $\mathbf{j}=0$. Here the parameter $\gamma=2 \tau_{\text {in }} \Delta(T) / \hbar$ is the product of the inelastic collision time $\tau_{\text {in }}$ for electron-phonon scattering, where $\Delta(T)$ $=4 k_{B} T_{c} u^{1 / 2} / \pi \sqrt{1-T / T_{c}}$ is the value of the order parameter at temperature $T$ which follows from Gor'kov's derivation ${ }^{25}$ of the Ginzburg-Landau equations. In Eqs. (1) and (2) all the physical quantities [order parameter $\psi=|\psi| \exp (i \phi)$, vector potential $\mathbf{A}$, and electrostatical potential $\varphi$ ] are measured in dimensionless units: the vector potential $\mathbf{A}$ and the momen- tum of the superconducting condensate $\mathbf{p}=\nabla \phi-\mathbf{A}$ are scaled by the unit $\Phi_{0} /(2 \pi \xi)$ (where $\Phi_{0}$ is the quantum of magnetic flux), the order parameter is in units of $\Delta_{0}$ and the coordinates are in units of the coherence length $\xi(T)$. In these units the magnetic field is scaled by $H_{c 2}=\Phi_{0} / 2 \pi \xi^{2}$ and the current density by $j_{0}=c \Phi_{0} / 8 \pi^{2} \lambda^{2} \xi$. Time is scaled in units of the Ginzburg-Landau relaxation time $\tau_{E}=4 \pi \sigma_{n} \lambda^{2} / c^{2}$ $=2 T \hbar / \pi \Delta_{0}^{2}$, the electrostatic potential $(\varphi)$ is in units of $\varphi_{0}$ $=c \Phi_{0} / 8 \pi^{2} \xi \lambda \sigma_{n}=\hbar / 2 e \tau_{\mathrm{GL}}$ (where $\sigma_{n}$ is the normal-state conductivity). Since we are in the regime where the width of the sample is much smaller than the effective penetration depth $W \ll \lambda^{2} / d$ we neglected the effect of the current-induced magnetic field and put $\mathbf{A}=(0, H x, 0)$ in Eqs. (1) and (2). The parameter $u$ is equal to 5.79 in accordance with Ref. 23. In our calculations we varied the other parameter $\gamma$ from 0 to 20 , which is proportional to the quasiparticle diffusion length $\lambda_{Q}$.

Superconductor-vacuum boundary conditions $\left.(\nabla-i \mathbf{A}) \psi\right|_{n}$ $=0,\left.\nabla \varphi\right|_{n}=0$ at the boundaries of the sample and the normal metal-superconductor boundary conditions $\psi=0$ and $\left.\nabla \varphi\right|_{n}=$ $-j$ at the contacts between the superconducting film with the normal leads are implemented in our simulations. To solve the system of Eqs. (1) and (2) we apply $s$ finite-difference representation for the order parameter and vector potential on a uniform Cartesian space grid and use the link variable approach $^{26}$ and standard iterative methods to find $\psi$. The electrostatic potential is obtained with the Fourier technique. When calculating the $I-V$ characteristics, we measured the voltage inside the superconducting sample [a distance $\delta$ away from the superconducting/normal $(\mathrm{S} / \mathrm{N})$ interface] as indicated in Fig. 1. In this way the contact resistance from the $\mathrm{S} / \mathrm{N}$ interface is not taken into account and our results correspond to a four-probe measurement.

\section{NUMERICAL RESULTS}

Let us first study the effect of the dimensions of the sample on the formation of the resistive state. Figure 2 shows the current-voltage $(I-V)$ characteristics of a superconducting stripe for $\gamma=20$ and for different values of the magnetic field. For zero magnetic field, a first PSL enters the sample at some critical current $j_{c 2}$ in the middle of the sample where the superconducting current reaches its maximum [inset 1 in Fig. 2(a)]. This phase-slip line leads to a finite jump in the $I-V$ curve [point 1 in Fig. 2(a)]. Contrary to phase-slip centers in one-dimensional superconductors, the oscillations of the order parameter are not necessary to be uniform along PSL: these oscillations may occur in the form of propagating waves carrying the order parameter singularities across the film. Such waves (named kinematic vortices) have been discovered in numerical simulations using the two-dimensional (2D) TDGL equations. ${ }^{17}$ It was shown that ${ }^{19}$ this phase-slip line, taken at an instantaneous time, consists of a line of counter propagating vortices and antivortices which are created on the opposite edge of the sample. These vortices and antivortices meet each other in the center of the sample where they annihilate. Averaged over time, this line of vortices appear as a phase-slip line. The critical current $j_{c 2}$ for the first phase-slip line is independent of the dimensions of 

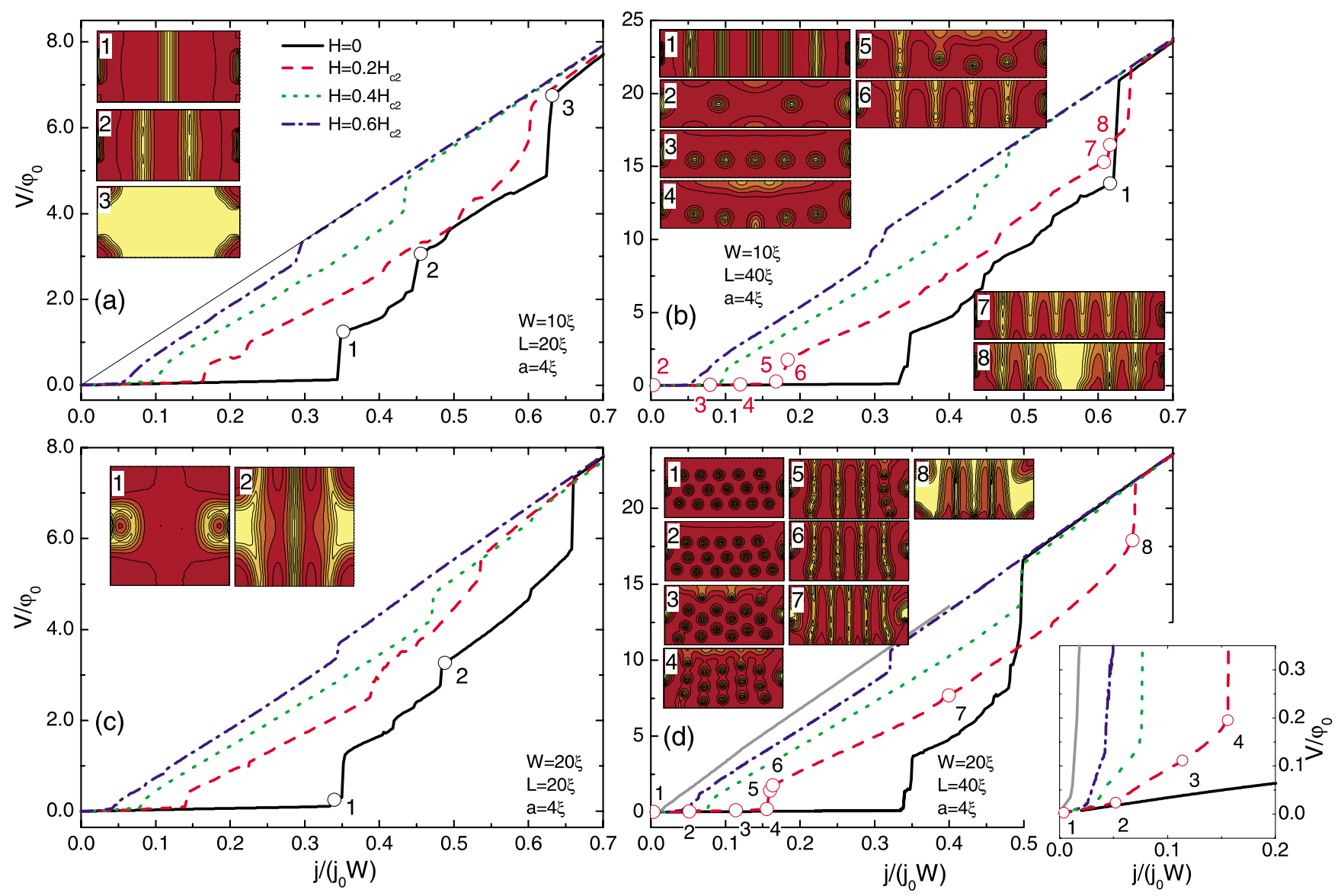

FIG. 2. (Color online) The $I-V$ characteristics of a superconducting strip obtained in the current increasing regime at the magnetic fields: $H=0$ (solid curve), $H=0.2 H_{c 2}$ (dashed curve), $H=0.4 H_{c 2}$ (dotted curve), and $H=0.6 H_{c 2}$ (dashed-dotted curve). The thin line in (a) indicates the $I-V$ curve of the sample in the normal state. The width of the sample is [(a) and (b)] $W=10 \xi$ and [(c) and (d)] W=20 $\xi$, the length is [(a) and (c) $L=20 \xi$ and [(b) and (d) $L=40 \xi$, and the size of the normal contact is $a=4 \xi$ and $\delta=5 \xi$. The insets show contour plots of the Cooper-pair density at a given time for the current values indicated in the main panel [red (dark gray) or light yellow (light gray) corresponds to high or zero Cooper-pair density]. Lower inset in (d) shows the lower part of the $I-V$ curve of the main panel.

the sample for the given $\gamma$ and equals to $j_{c 2} /\left(j_{0} W\right) \simeq 0.38$. After the appearance of the first phase-slip line the superconducting current in its neighborhood is strongly suppressed over a distance of about the penetration depth $\lambda_{Q}$ of the normal current (electric field) in the superconductor. As a result one needs a larger current to reach the condition for the nucleation of the next phase-slip line in the strip. In general, the possible number of active PSLs is equal to the number of voltage steps in the corresponding $I-V$ curve. Due to the symmetry of the sample more than one PSLs can penetrate the sample at the same time and tend to avoid those already in place [inset 2 in Fig. 2(a)]. The number of those highly dissipative domains increases with increasing the sample dimensions [inset 1 in Fig. 2(b)]. Because of the close proximity of the normal contacts superconductivity is locally suppressed [inset 1 in Fig. 2(c)] and phase-slip lines can also be distorted [inset 1 in Fig. 2(c)]. With further increasing the applied current the system transits to a highly dissipative state with a normal path between the contacts [inset 3 in Fig. 2(a)]. However, the resistance of this state can be different from the one of the normal state [thin dotted line in Fig. 2(a)] because superconductivity is preserved at the corners of the sample [see inset 3 of Fig. 2(a)].
When we apply a magnetic-field vortices penetrate the sample and arrange themselves into a vortex chains in narrow samples [inset 2 in Fig. 2(b)] or into a triangular lattice for larger samples [inset 1 in Fig. 2(d)]. As the applied current increases the surface barrier for vortices to enter the sample decreases and consequently, the number of vortices inside the sample can increase with increasing $j$ [compare insets 2 and 3 in Fig. 2(b)]. At the same time vortex lattice shifts as a whole due to the Lorentz of the current [inset 2 in Fig. 2(d)]. With further increasing current many vortices are set into motion and they penetrate the sample from one side and exit from the other side of the sample [inset 3 in Fig. $2(d)]$. This motion of vortices leads to a smaller voltage jump at $j=j_{c 2}(H)$ which is much smaller than the voltage jump at zero magnetic field [see the lower inset in Fig. 2(d)]. At the same time a rearrangement of the vortex lattice takes place and vortex rows are formed [inset 4 in Fig. 2(d)]. With further increasing current vortex channels, the phase-slip lines appear in the sample [inset 5 in Fig. 2(b)]. The coexistence of fast and slow moving vortices is also possible [inset 5 in Fig. 2(d)]. This PSL is the 2D analog of the phase-slip centers in superconducting wires, ${ }^{27}$ with the difference that here the order parameter may vary across the $2 \mathrm{D}$ wire. ${ }^{28}$ The 


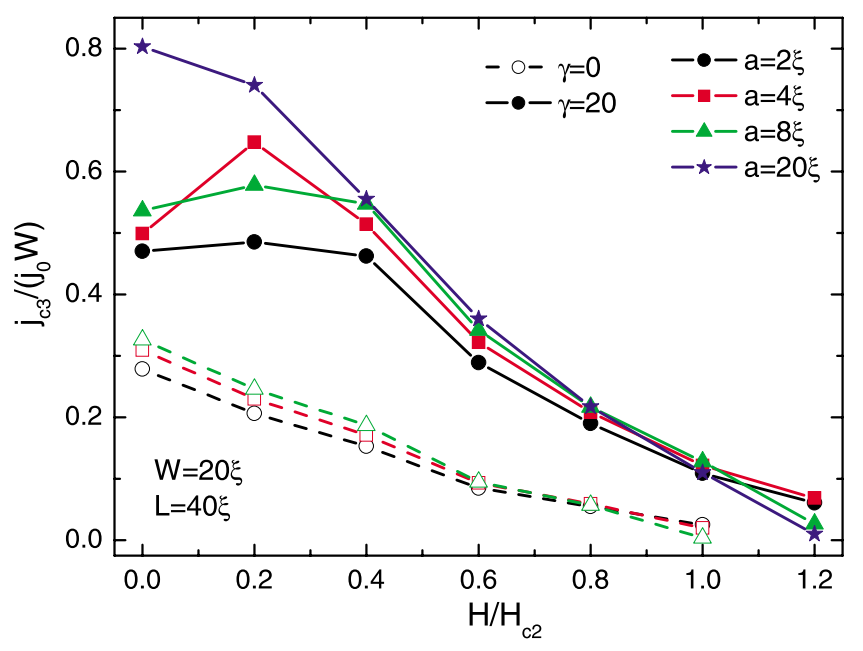

FIG. 3. (Color online) Superconducting normal transition current density $j_{c 3}$ as a function of the applied field $H / H_{c 2}$ for different widths of the normal contacts $a$. Solid symbols represent the results obtained for $\gamma=0$ and open symbols are the results for $\gamma=20$. The size of the sample is $L=40 \xi$ and $W=20 \xi$.

physical reason for the appearance of these vortex lines is as follows: the moving vortices leave behind a wake of reduced $|\psi|$ because of nonequilibrium effects, ${ }^{18,19}$ i.e., the condensate at a particular point needs a finite time to recover to its initial value after the passage of a vortex. This induces a short-range vortex attraction along the wire on top of the normal isotropic vortex-vortex repulsion, resulting into an anisotropic effective vortex-vortex interaction. With further increasing the current more PSLs enter the sample [inset 7 in Figs. 2(b) and 2(d)], in some cases interpenetrating the previous ones. Therefore, the steps in the $I-V$ curve become smaller as compared to the zero-magnetic-field case. Before the system transits to the normal-state individual vortex channels start merging [inset 8 in Figs. 2(b) and 2(d)].

For longer samples we found the very interesting result that the superconducting (resistive) to normal-state transition critical current density $j_{c 3}$ increases with applying weak magnetic field [compare solid black and dashed curves in Figs. 2(b) and 2(d)] - the system is in the phase-slip line state for larger values of the applied current. This effect disappears with further increasing the applied field. Shorter samples sustain larger applied currents at zero magnetic field [see Figs. 2(a) and 2(c)]. To convince us that this result is not an accident we studied the found phenomena in more detail. Figure 3 shows the value of the current at which point the system is driven to the normal state, i.e., the upper critical current density $j_{c 3}$ as a function of the applied magnetic field. For $\gamma=0$ (dashed lines and open symbols), $j_{c 3}$ is a monotonically decreasing function of $H$ for all values of $a . j_{c 3}$ is larger for larger size of the normal contacts $a$. For larger $\gamma$ values (solid lines) there is a maximum in the $j_{c 3}(H)$ curve. The height of this maximum is largest for intermediate values of $a$ and vanishes for smaller and larger size of the normal contacts.

It turns out that the increase in $j_{c 3}$ with magnetic field is caused by the nonuniform distribution of currents in the sample due to the presence of normal contacts. To support

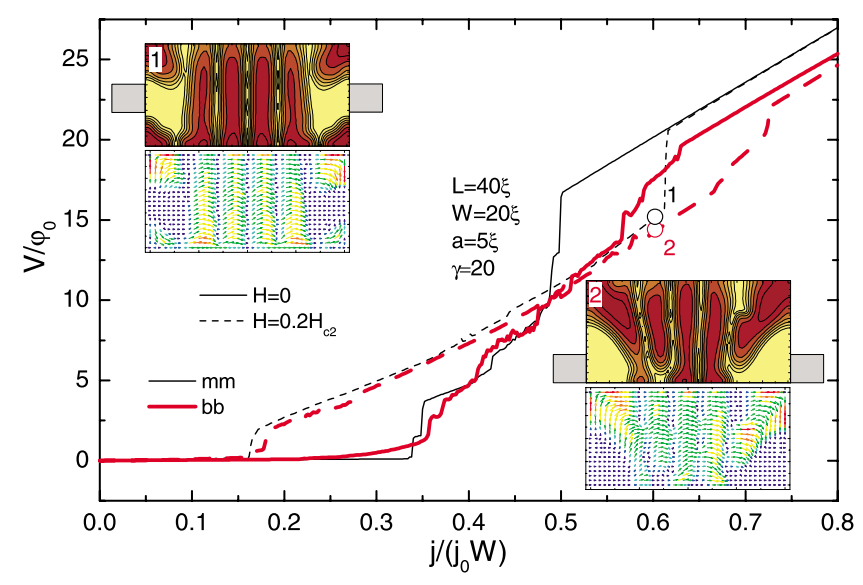

FIG. 4. (Color online) The $I-V$ curve of a superconducting stripe ( $W=20 \xi, L=40 \xi$, and $\gamma=20$ ) for $H=0$ (solid curves) and $H$ $=0.2 H_{c 2}$ (dashed curves). The normal contacts $(a=5 \xi)$ are attached in the middle of the sample side (thin curves) and on the bottom of the sample side (thicker curves). Insets show the Cooper-pair density plots (top figures) and vector plots of the superconducting current (bottom figures) for $j /\left(j_{0} W\right)=0.58$, when the contacts are in the middle of the sample side (inset 1) and on the bottom of the sample side (inset 2).

this idea we also conducted simulations for the case where the normal contacts are shifted to the bottom of the lateral size of the sample as illustrated in the second inset of Fig. 4 ( $b b$ sample). Figure 4 shows the $I$ - $V$ curves of the $b b$ sample and the sample with contacts attached in the middle of lateral size $\mathrm{mm}$ sample $H=0$ (solid curves) and $H=0.2 H_{c 2}$ (dashed curves). As we see from this figure, $j_{c 3}$ for both values of the applied field is considerably increased in the $b b$ sample, where a strongly nonuniform distribution of the supercurrents is found (see the insets in Fig. 4). The latter means that the nonuniform distribution of currents plays an important role in the formation of a stable resistive state.

We have the following explanation for found phenomena. It is well known (see, for example, Ref. 28) that in superconductors with a uniform distribution of the current density over the cross section of the sample the position of the normal metal-superconductor boundary is unstable. If the current density exceeds some critical value $j_{c}$ then the normal region expands and for $j<j_{c}$ the superconducting region grows. ${ }^{29}$ In the framework of Eqs. (1) and (2) the critical current $j_{c} \simeq \sqrt{\gamma / u}$ (for $\gamma \gg 1$ ). ${ }^{30}$ In our model system with width of the current-injected contacts $a<W$ we have a nonuniform distribution of the current density over the length of the stripe even in the normal state. The current density is maximal near the current-injected contacts and it can be much less in the rest of the sample (if $a \ll W$ ). Therefore, when the local current density exceeds $j_{c}$ the N-S boundary moves deep into the superconductor and should stop when the current density at the N-S boundary becomes smaller than $j_{c}$. Such a situation takes place in case of $a<W$ and one may suppose that the length of the N-S boundary may increase up to the width of the sample. This would mean that the total critical current density $j_{c 3}$ should be the same for different widths $a$ which is not observed. Our calculations shows that $j_{c 3}$ monotonically increases with increasing $a$ (at 

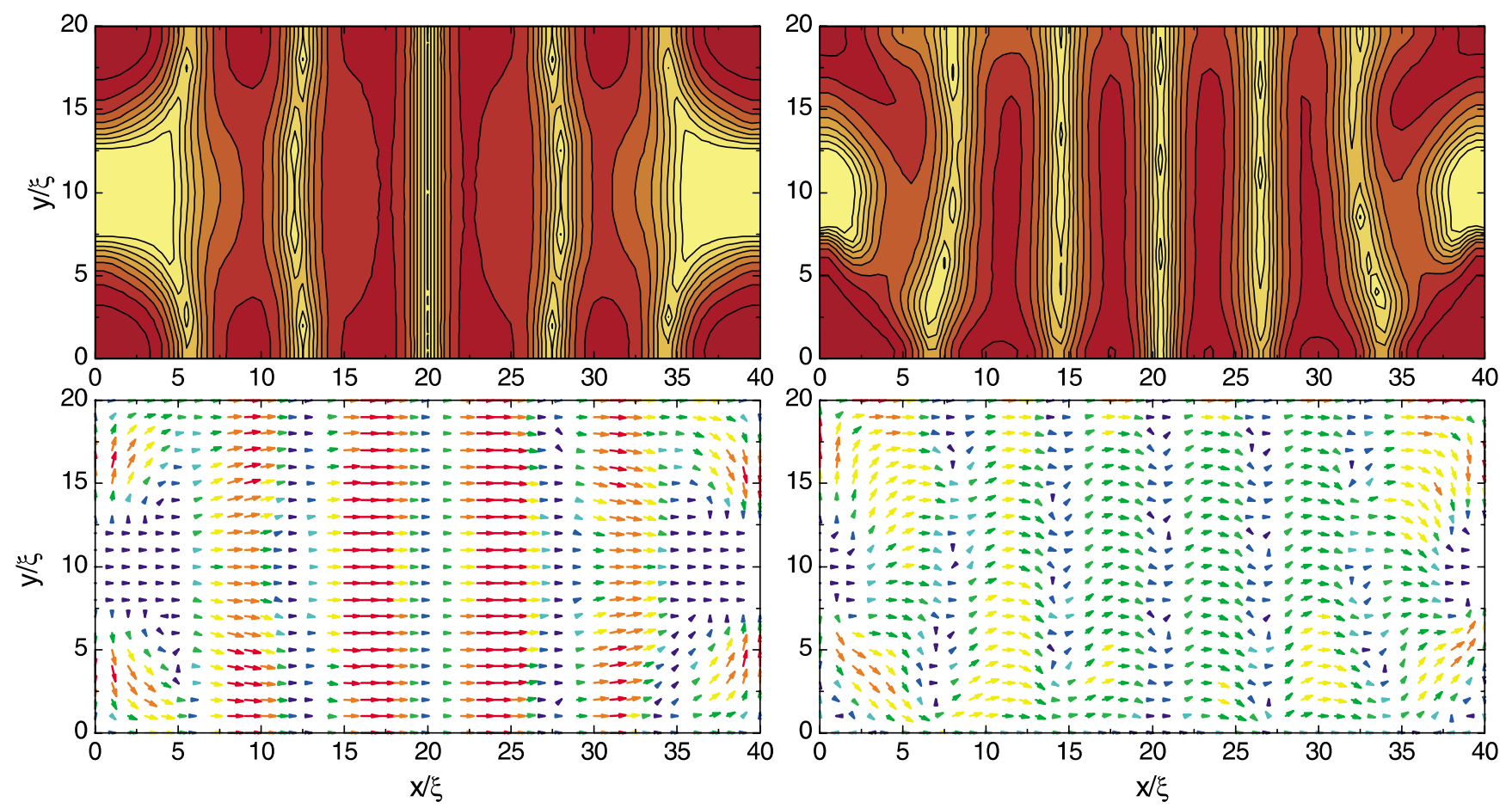

FIG. 5. (Color online) [(a) and (b)] Contour plots of the Cooper-pair density and [(c) and (d)] vector plots of the superconducting current density of the sample with $L=40 \xi, W=20 \xi$, and $a=4 \xi$ for $j /\left(j_{0} W\right)=0.49$ and for [(a) and (c) $H=0$ and $[(\mathrm{b})$ and (d) $] H=0.2 H_{c 2}$.

$H=0$ ) and reaches the maximal value at $a=W$ (see Fig. 3). Therefore the above picture of stability of N-S boundary in case of nonuniform current density distribution is clearly an oversimplification. Probably the nonuniformity plays an important role because we found that the length scale over which the current density becomes uniform is equal to the width of the stripe (if $a \ll W$ ). Hence the above picture works only for $L \gg W$ and for $L \sim W$ the finite length of the stripe should be taken into account (we observed an increase in the critical current $j_{c 3}$ with increasing $L$ at fixed $a<W$ ). Indeed, for short stripes (with $L \sim W$ ) the current density in the middle of the stripe is always larger than the current density at the boundary of the stripe of the same length but with $a$ $=W$. When $a$ approaches $W$ the above-mentioned characteristic length scale gradually decreases and it goes to zero when $a=W$. This can explain the results shown in Fig. 3 for $H=0$.

When we apply a small magnetic field the current density distribution is changed [see Fig. 5(c)]. Screening currents appear, which are perpendicular to the transport current, at the sides of the stripe where the current is injected. It leads to a deformation of the NS boundary and of the shape of the phase-slip lines [Figs. 5(a) and 5(b)]. In case of $H>0$ the curvature of the phase-slip lines changes due to the repulsion of the vortices, which move along the phase-slip lines.

\section{CONCLUSIONS}

To conclude, using the phenomenological TDGL formalism, we studied the transport properties of thin superconducting stripes with attached normal leads in the presence of a perpendicular magnetic field. We found that at zero magnetic field a finite resistance is induced by phase-slip lines. As the parameter $\gamma$ increases the current range, over which such phase-slip state is possible, increases to larger and lower currents. Consequently, the superconducting normal transition current $j_{c 3}$ increases with increasing $\gamma$. But for $\gamma=0$ no such oscillatory phase-slip solution exists at zero field. When a magnetic field is applied to the system, the number of distinct discontinuities in the $I-V$ curve increases. This behavior is consistent with the restabilization of a moving vortex distribution by the formation of a dynamic vortex phase with distortion in the local flux density and vortex velocity. When the critical velocity of vortices is reached phase-slip lines appear across the sample. This phase-slip mechanism turns out to be more effective in producing resistance. The $I-V$ curve exhibits a hysteretic behavior and the size of this hysteresis decreases with increasing magnetic field.

The most interesting result of our study is that for larger values of $\gamma$ the normal-state transition current $j_{c 3}$ is increased in a small external magnetic field as compared to $j_{c 3}$ at zero magnetic field. The reason for such magnetic-field-induced increase in the critical current is the nonuniform distribution of currents along the sample (due to the presence of normal contacts), which keeps the system in a resistive state for larger values of the applied current.

\section{ACKNOWLEDGMENTS}

This work was supported by the Flemish Science Foundation (FWO-Vl) and the Belgian Science Policy (IAP). D.Y.V. acknowledges support from the Dynasty Foundation. G.R.B. acknowledges support from FWO-Vlaanderen. 
*francois.peeters@ua.ac.be

${ }^{1}$ A. I. Larkin and Yu. N. Ovchinnikov, Zh. Eksp. Teor. Fiz. 68, 1915 (1975) [Sov. Phys. JETP 41, 960 (1976)]; Nonequilibrium Superconductivity, edited by D. N. Langenberg and A. I. Larkin (North-Holland, Amsterdam, 1986), p. 493.

${ }^{2}$ L. E. Musienko, I. M. Dmitrenko, and V. G. Volotskaya, Zh. Pis'ma Eksp. Teor. Fiz. 31, 603 (1980) [JETP Lett. 31, 567 (1980)].

${ }^{3}$ W. Klein, R. P. Huebener, S. Gauss, and J. Parisi, J. Low Temp. Phys. 61, 413 (1985).

${ }^{4}$ D. Babic, J. Bentner, C. Surgers, and C. Strunk, Phys. Rev. B 69, 092510 (2004).

${ }^{5}$ M. Tian, J. Wang, J. S. Kurtz, Y. Liu, M. H. W. Chan, T. S. Mayer, and T. E. Mallouk, Phys. Rev. B 71, 104521 (2005).

${ }^{6}$ A. V. Samoilov, M. Konczykowski, N. C. Yeh, S. Berry, and C. C. Tsuei, Phys. Rev. Lett. 75, 4118 (1995).

${ }^{7}$ S. G. Doettinger, R. P. Huebener, R. Gerdemann, A. Kuhle, S. Anders, T. G. Trauble, and J. C. Villegier, Phys. Rev. Lett. 73, 1691 (1994).

${ }^{8}$ S. G. Doettinger, S. Kittelberger, R. P. Huebener, and C. C. Tsuei, Phys. Rev. B 56, 14157 (1997).

${ }^{9}$ Z. L. Xiao and P. Ziemann, Phys. Rev. B 53, 15265 (1996).

${ }^{10}$ Z. L. Xiao, P. Voss-de Haan, G. Jakob, T. Kluge, P. Haibach, H. Adrian, and E. Y. Andrei, Phys. Rev. B 59, 1481 (1999).

${ }^{11}$ A. E. Koshelev and V. M. Vinokur, Phys. Rev. Lett. 73, 3580 (1994).

${ }^{12}$ J. A. Fendrich, U. Welp, W. K. Kwok, A. E. Koshelev, G. W. Crabtree, and B. W. Veal, Phys. Rev. Lett. 77, 2073 (1996).

${ }^{13}$ Z. L. Xiao, E. Y. Andrei, and P. Ziemann, Phys. Rev. B 58, 11185 (1998).

${ }^{14}$ M. N. Kunchur, Phys. Rev. Lett. 89, 137005 (2002).

${ }^{15}$ S. V. Lempitskii, Zh. Eksp. Teor. Fiz. 90, 793 (1986) [Sov. Phys.
JETP 63, 462 (1986).

${ }^{16}$ A. Weber and L. Kramer, J. Low Temp. Phys. 84, 289 (1991).

${ }^{17}$ A. Andronov, I. Gordion, V. Kurin, I. Nefedov, and I. Shereshevsky, Physica C 213, 193 (1993).

${ }^{18}$ L. I. Glazman, Fiz. Nizk. Temp. 12, 688 (1986) [Sov. J. Low Temp. Phys. 12, 389 (1986)].

${ }^{19}$ D. Y. Vodolazov and F. M. Peeters, Phys. Rev. B 76, 014521 (2007).

${ }^{20}$ M. Tian, N. Kumar, S. Xu, J. Wang, J. S. Kurtz, and M. H. W. Chan, Phys. Rev. Lett. 95, 076802 (2005); M. Tian, N. Kumar, J. Wang, S. Xu, and M. H. W. Chan, Phys. Rev. B 74, 014515 (2006).

${ }^{21}$ D. Y. Vodolazov, Phys. Rev. B 75, 184517 (2007).

${ }^{22}$ V. A. Schweigert, F. M. Peeters, and P. S. Deo, Phys. Rev. Lett. 81, 2783 (1998); V. A. Schweigert and F. M. Peeters, Phys. Rev. B 57, 13817 (1998).

${ }^{23}$ L. Kramer and R. J. Watts-Tobin, Phys. Rev. Lett. 40, 1041 (1978).

${ }^{24}$ R. J. Watts-Tobin, Y. Krähenbühl, and L. Kramer, J. Low Temp. Phys. 42, 459 (1981).

${ }^{25}$ L. P. Gor'kov, Zh. Eksp. Teor. Fiz. 36, 1918 (1959) [Sov. Phys. JETP 9, 1364 (1959)].

${ }^{26}$ R. Kato, Y. Enomoto, and S. Maekawa, Phys. Rev. B 47, 8016 (1993).

${ }^{27}$ W. J. Skocpol, M. R. Beasley, and M. Tinkham, J. Low Temp. Phys. 16, 145 (1974).

${ }^{28}$ D. Y. Vodolazov, B. J. Baelus, and F. M. Peeters, Physica C 404, 400 (2004).

${ }^{29}$ B. I. Ivlev and N. B. Kopnin, Sov. Phys. Usp. 27, 206 (1984) [Usp. Fiz. Nauk 142, 435 (1984)].

${ }^{30}$ D. Y. Vodolazov, A. Elmuradov, and F. M. Peeters, Phys. Rev. B 72, 134509 (2005). 